\title{
Application of vibrational power flow to a passenger car for reduction of interior noise
}

\author{
S.K. Lee \\ Department of Mechanical Engineering, Inha \\ University, Yong Hyun Dong 253, Inchon, Korea \\ 402-751 \\ Tel.: +8232860 7305; Fax: +82 32868 1716; \\ E-mail: sangkwon@inha.ac.kr
}

Received 27 August 1999

Revised 31 May 2000

Reduction of structure-borne noise in the compartment of a car is an important task in automotive engineering. Transfer path analysis using the vibroacoustic reciprocity technique or multiple path decomposition method has generally been used for structure-borne noise path analysis. These methods are useful for solving a particular problem, but they do not quantify the effectiveness of vibration isolation of each isolator of a vehicle. To quantify the effectiveness of vibration isolation, vibrational power flow has been used for a simple isolation system or a laboratory-based isolation system. It is often difficult to apply the vibrational power flow technique to a complex isolation system like a car. In this paper, a simple equation is derived for calculation of the vibrational power flow of an isolation system with multiple isolators such as a car. It is successfully applied not only to quantifying the relative contributions of eighteen isolators, but also to reducing the structure-borne noise of a passenger car. According to the results, the main contributor of the eighteen isolators is the rear roll mount of an engine. The reduced structure-borne noise level is about $5 \mathrm{dBA}$.

\section{Introduction}

Many components of cars are mounted in relatively flexible seating structures such as the underbody structure. Compliant vibration isolators are often used to reduce the transmission of unwanted vibration from an engine to seating structures. However, despite isolation, vibration transmission problems in automotive engineering sometimes still remain, particularly in complex installations with more stringent requirements for vibration and noise levels, combined with a trend to lighter car bodies and more powerful engines. Especially in a competitive automotive market, there is obviously the need to reduce the noise level in the compartment to the lowest possible level within a given cost constraint. Such a procedure would ostensibly involve some sort of structure-borne transmission path analysis to assess the relative noise contributions. This can be done at present by using a reciprocity technique [7] or multiple path decomposition method [2]. These methods are useful in solving particular problems but do not quantify the effectiveness of vibration isolation of each isolator of a vehicle. Pinnington [5] did a good job in explaining the vibrational power flow technique as a method to quantify the effectiveness of a vibration isolator and its application in a mechanical system. Before this, Goyder [1] introduced the concept of applying point mobility to the calculation of the vibrational power transmitted from a vibrating machine to its seating structure. Pinnington [4] then investigated the parameters controlling power transmission from a vibrating machine to the seating structure for the isolated system with a single isolator. Recently, Pinnington conducted research about transient vibrational flow [6]. Petersson [3] showed a practical method for applying vibrational power flow to predict the structure-borne sound transmission in a diesel generator system. In this paper, a simple equation is derived for the calculation of the vibrational power for an isolator system with multiple isolators like a car. It is also successfully applied to identify the major isolator contribution to the increase of structure-borne noise in a passenger car. Eighteen isolators of a test vehicle are considered as vibration transmission paths: the engine mount, the gearbox mount, the exhaust mount, etc. By evaluation of the vibrational power flow of each isolator, it is found that the real roll mount of the engine is a major path, and its reduced stiffness results in the reduction of the booming noise of up to 5dBA around $1800 \mathrm{rpm}$.

\section{Theory of vibrational power flow}

Power, which is the rate at which work is done, is given by the relationship [1] 


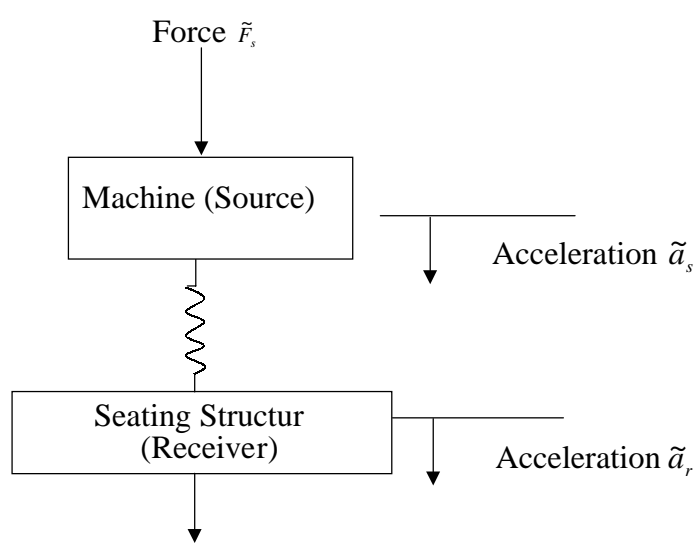

Force $\tilde{F}_{r}$

Fig. 1. Geometry for calculation of power flow through an isolator.

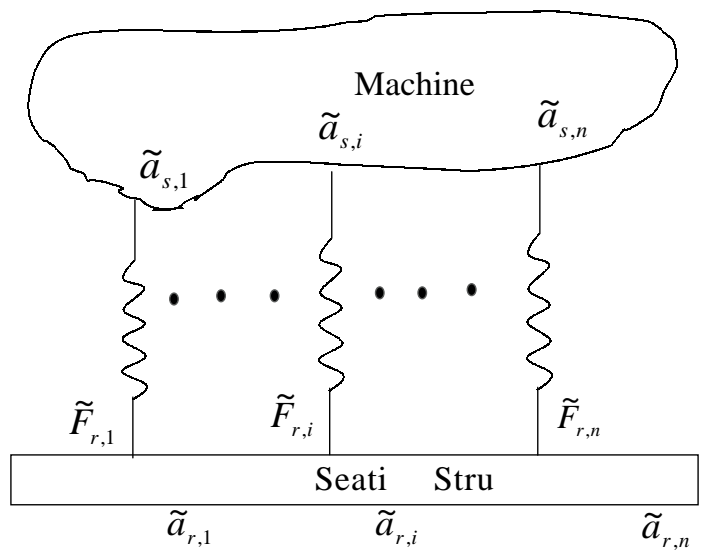

Fig. 2. Geometry for calculation of power flow through multiple isolators.

$$
P_{i}=F_{i} V_{i}
$$

where $F_{i}$ and $V_{i}$ are the instantaneous values of force and velocity at a point. When vibrational power flows through an isolator it is necessary to consider it as an intensity and therefore with the force $F_{i}$ determined as a stress. With a vibrating structure the time-averaged vibrational flow of power is more important than the instantaneous value, and when force and velocity are harmonic this is given by

$$
P=\frac{1}{T} \int_{0}^{T} F_{i} V_{i} d t
$$

A force with a harmonic time dependence $F_{i}=$ $\tilde{F} e^{j \omega t}$ acting at a point on a structure of mobility $\tilde{M}=|M| e^{j \phi}$ causes a velocity of $V_{i}=\tilde{V} e^{j \omega t}$ at that point, where $\tilde{F}$ and $\tilde{V}$ are complex. The time averaged vibrational power is given by $P=\frac{1}{2}|F||V| \cos \phi$, or

$$
P=\frac{1}{2} \operatorname{Re}\{\tilde{F} * \tilde{V}\}=\frac{1}{2} \operatorname{Re}\{\tilde{F} \tilde{V} *\}
$$

where $\phi$ is the relative phase angle and $*$ denotes the complex conjugate. When the acceleration on a structure is used instead of a velocity, the time-averaged power, Eq. (3) is rewritten as follows:

$$
P=-\frac{1}{2 \omega} \operatorname{Im}\{\tilde{F} \tilde{a} *\}
$$

where $\tilde{a}=j \omega \tilde{V}$. Therefore, the time-averaged vibrational power transmitted through a single isolator, as shown in Fig. 1, is calculated by a product of the force acting at a point on a seating structure (receiver) and the acceleration at that point. This force can also be calculated by using apparent mass as follows:

$$
\tilde{F}_{r}=\frac{\tilde{F}_{r}}{\tilde{a}_{r}} \tilde{a}_{r}+\frac{\tilde{F}_{r}}{\tilde{a}_{s}} \tilde{a}_{s}=\tilde{M}_{r r} \tilde{a}_{r}+\tilde{M}_{r s} \tilde{a}_{s}
$$

where $\tilde{M}_{r r}$ and $\tilde{M}_{r s}$ are the point and transfer apparent mass, respectively, $\tilde{F}_{r}$ is the force at the seating structure (receiver), and $\tilde{a}_{s}$ and $\tilde{a}_{r}$ are the accelerations on the machine (source) and the seating structure of an isolation system as shown in Fig. 1. Thus, using Eq. (4), the vibrational power flow on a seating structure is obtained by

$$
P=-\frac{1}{2 \omega} \operatorname{Im}\left\{\tilde{M}_{r s} \tilde{a}_{s} \tilde{a}_{r}^{*}+\tilde{M}_{r r} \tilde{a}_{r} \tilde{a}_{r}^{*}\right\}
$$

If an isolator is a natural rubber with damping, the transfer apparent mass is simply assumed by

$$
\tilde{M}_{r s}=\frac{K}{\omega^{2}}+j \eta \frac{K}{\omega^{2}}
$$

where $K$ is the dynamic stiffness and $\eta$ is the loss factor of an isolator. Similarly, the time-averaged vibrational power transmitted through two single isolators can also be obtained by using the following two forces:

$$
\begin{aligned}
\tilde{F}_{r, 1}= & \frac{\tilde{F}_{r, 1}}{\tilde{a}_{r, 1}} \tilde{a}_{r, 1}+\frac{\tilde{F}_{r, 1}}{\tilde{a}_{s, 1}} \tilde{a}_{s, 1} \\
& +\frac{\tilde{F}_{r, 1}}{\tilde{a}_{r, 2}} \tilde{a}_{r, 2}+\frac{\tilde{F}_{r, 1}}{\tilde{a}_{s, 2}} \tilde{a}_{s, 2} \\
= & \tilde{M}_{r r, 11} \tilde{a}_{r, 1}+\tilde{M}_{r s, 11} \tilde{a}_{s, 1} \\
& +\tilde{M}_{r s, 12} \tilde{a}_{r, 2}+\tilde{M}_{r s, 12} \tilde{a}_{s, 2} \\
= & \tilde{M}_{r r, 11} \tilde{a}_{r, 1}+\tilde{M}_{r r, 12} \tilde{a}_{r, 2} \\
& +\tilde{M}_{r s, 11} \tilde{a}_{s, 2}+\tilde{M}_{r s, 12} \tilde{a}_{s, 2}
\end{aligned}
$$

and 


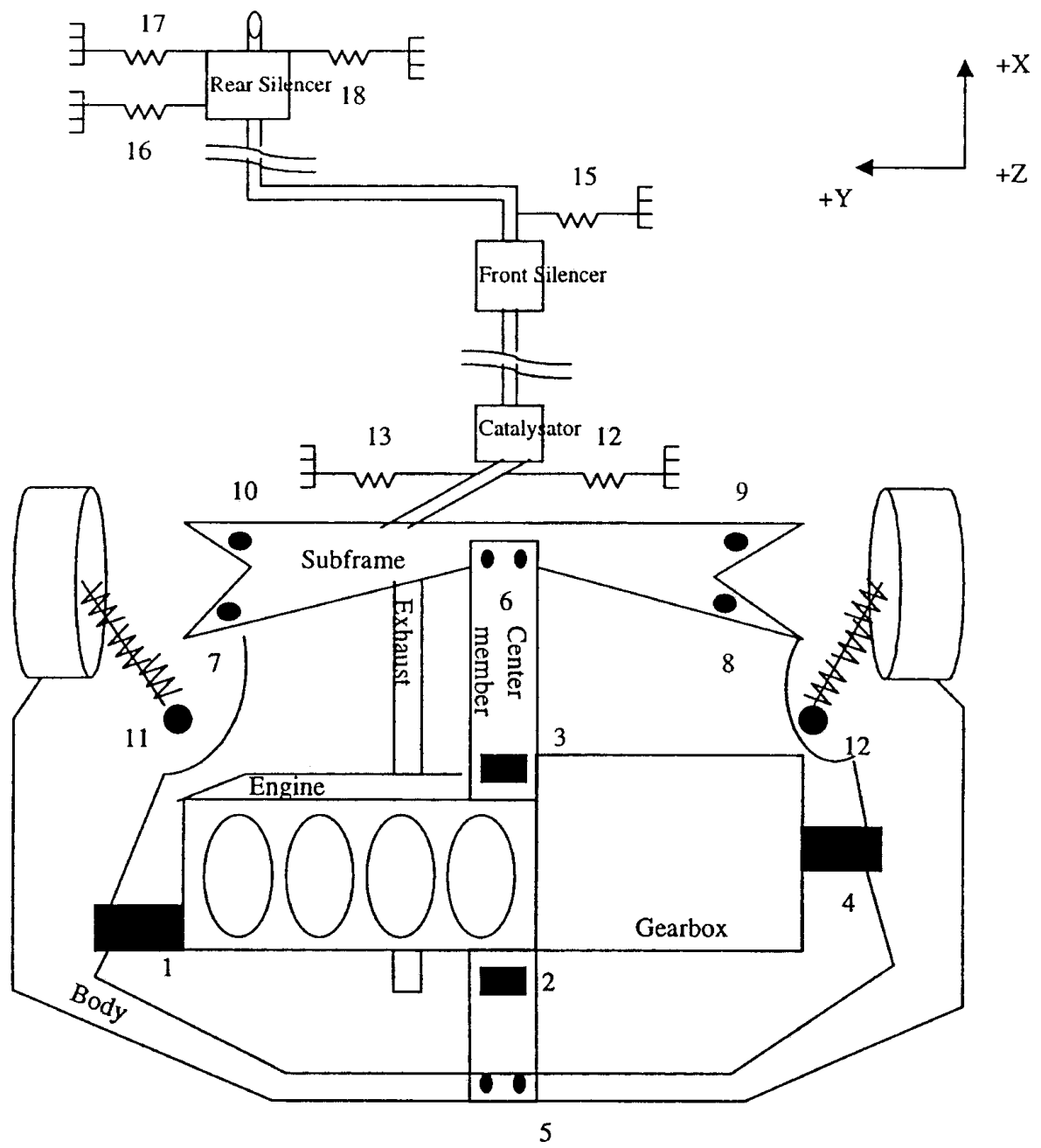

Fig. 3. Layout for eighteen isolators of the test vehicle.

$$
\begin{aligned}
\tilde{F}_{r, 2}= & \frac{\tilde{F}_{r, 2}}{\tilde{a}_{r, 1}} \tilde{a}_{r, 1}+\frac{\tilde{F}_{r, 2}}{\tilde{a}_{s, 1}} \tilde{a}_{s, 1} \\
& +\frac{\tilde{F}_{r, 2}}{\tilde{a}_{r, 2}} \tilde{a}_{r, 2}+\frac{\tilde{F}_{r, 2}}{\tilde{a}_{s, 2}} \tilde{a}_{s, 2} \\
= & \tilde{M}_{r r, 21} \tilde{a}_{r, 1}+\tilde{M}_{r s, 21} \tilde{a}_{s, 1} \\
& +\tilde{M}_{r s, 22} \tilde{a}_{r, 2}+\tilde{M}_{r s, 22} \tilde{a}_{s, 2} \\
= & \tilde{M}_{r r, 21} \tilde{a}_{r, 1}+\tilde{M}_{r r, 22} \tilde{a}_{r, 2} \\
& +\tilde{M}_{r s, 21} \tilde{a}_{s, 2}+\tilde{M}_{r s, 22} \tilde{a}_{s, 2}
\end{aligned}
$$

where the subscripts 1 and 2 denote the different isolators, 1 and 2. For the isolation system with $n$ isolators as shown in Fig. 2, the vibrational power flow transmitted through $n$ isolators is obtained by using a force vector,

$$
\{\tilde{F}\}_{r}=[\tilde{M}]_{r s}\{\tilde{a}\}_{s}+[\tilde{M}]_{r r}\{\tilde{a}\}_{r}
$$

where

$$
\begin{aligned}
& \{\tilde{F}\}_{r}=\left\{\begin{array}{c}
\tilde{F}_{r, 1} \\
\vdots \\
\tilde{F}_{r, n}
\end{array}\right\},\{\tilde{a}\}_{r}=\left\{\begin{array}{c}
\tilde{a}_{r, 1} \\
\vdots \\
\tilde{a}_{r, n}
\end{array}\right\} \\
& \{\tilde{a}\}_{s}=\left\{\begin{array}{c}
\tilde{a}_{s, 1} \\
\vdots \\
\tilde{a}_{s, n}
\end{array}\right\}
\end{aligned}
$$

and

$$
[\tilde{M}]_{r r}=\left[\begin{array}{ccc}
\tilde{M}_{r r, 11} & \cdots & \tilde{M}_{r r, 1 n} \\
\vdots & \ddots & \vdots \\
\tilde{M}_{r r, n 1} & \cdots & \tilde{M}_{r r, n n}
\end{array}\right]
$$




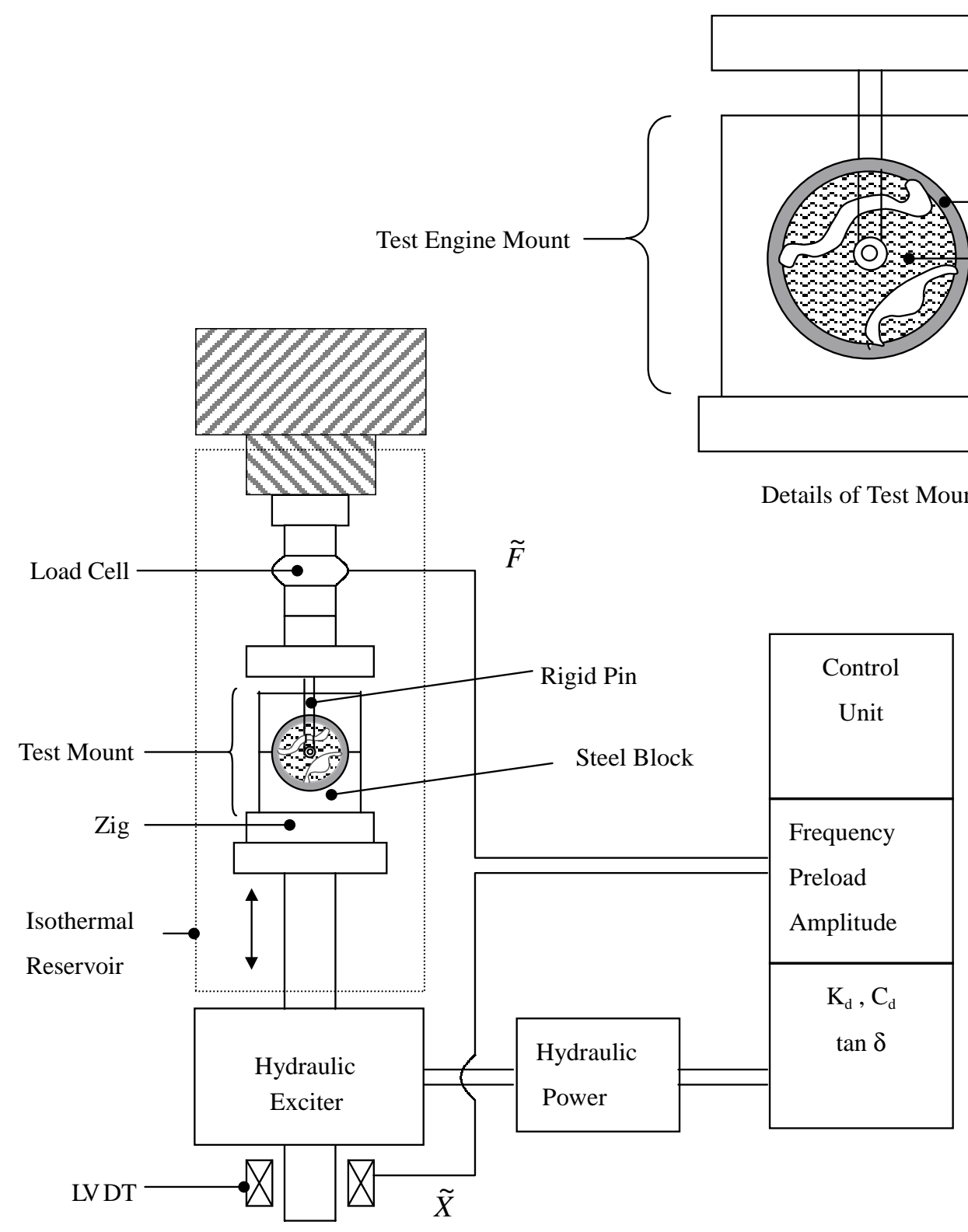

Fig. 4. Elastomer testing system for measurement of complex stiffness.

$$
[\tilde{M}]_{r s}=\left[\begin{array}{ccc}
\tilde{M}_{r s, 11} & \cdots & \tilde{M}_{r s, 1 n} \\
\vdots & \ddots & \vdots \\
\tilde{M}_{r s, n 1} & \cdots & \tilde{M}_{r s, n n}
\end{array}\right]
$$

In Eq. (10), $\{\tilde{a}\}_{s}$ and $\{\tilde{a}\}_{r}$ are the acceleration vectors on the machine and the seating structure, respectively, and $[\tilde{M}]_{r r}$ and $[\tilde{M}]_{r s}$ are the apparent mass matrix of a seating structure and the transfer apparent mass matrix between the machine and the seating structure, respectively. Therefore, a simple form of the time-averaged vibrational power flow $P$ for an isolation system with $n$ isolators is calculated as follows:

$$
\begin{aligned}
P= & -\frac{1}{20} \operatorname{Im}\left\{\{\tilde{a}\}_{s}^{T}[\tilde{M}]_{r s}^{T}\{\tilde{a}\}_{r}^{*}\right. \\
& \left.+\{\tilde{a}\}_{r}^{T}[\tilde{M}]_{r r}^{T}\{\tilde{a}\}_{r}^{*}\right\}
\end{aligned}
$$

However, Eq. (11) can give only the total power absorbed by the seating structure, implying that this method cannot be used to discriminate between the power from the machine in question, airborne noise, or 

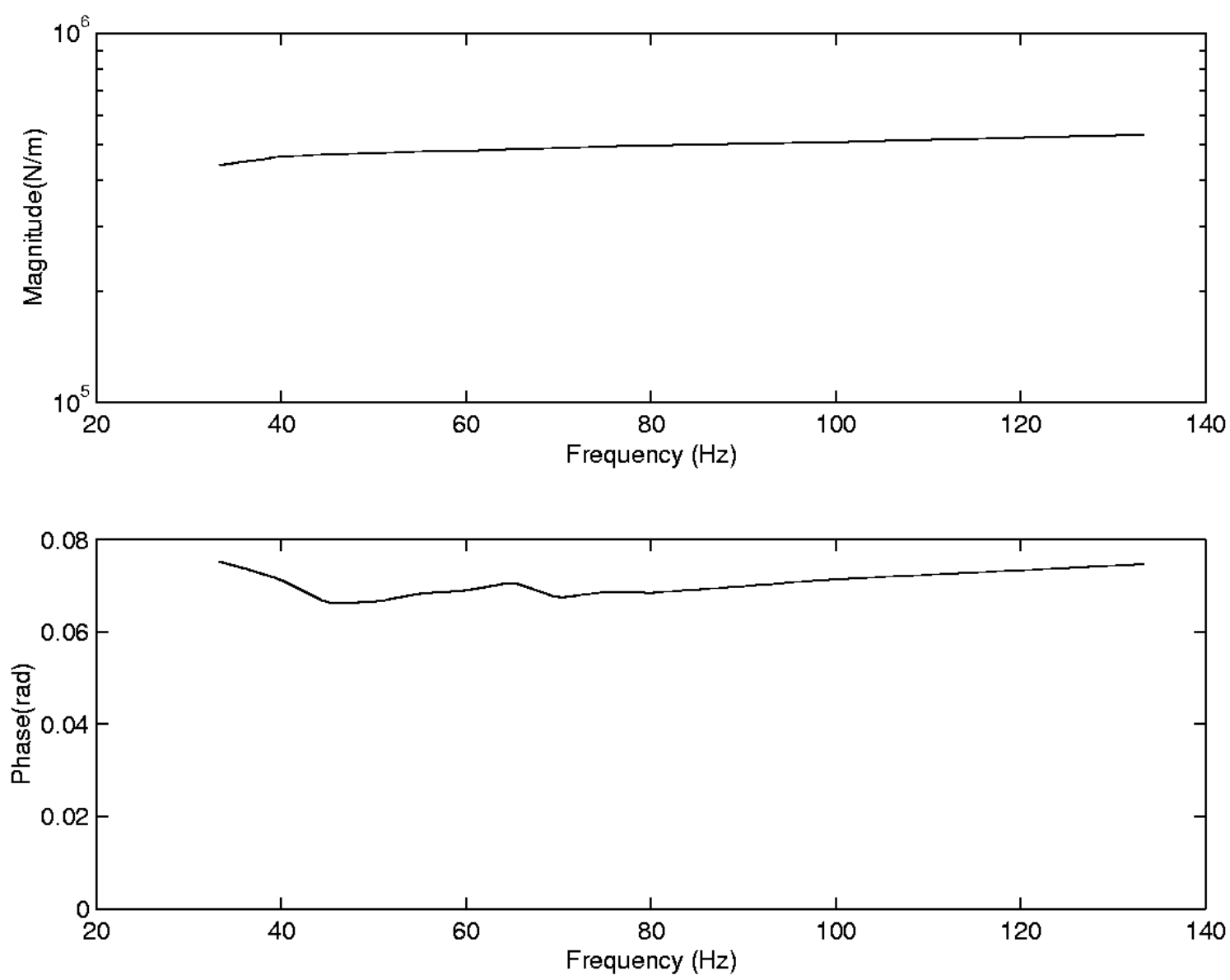

Fig. 5. Measured complex dynamic stiffness of the rear roll mount.

an adjacent machine. In contrast to measuring the total power input to the seating structure, mentioned above, the vibrational power transmitted by each individual isolator can be measured independently of other vibration sources. From Eq. (5), for a vibrating machine, the imaginary part of the point apparent mass $\tilde{M}_{r r}$ generally is less than that of the transfer apparent mass $\tilde{M}_{r s}$ and the acceleration $\tilde{a}_{r}$ of the seating structure is less than the acceleration $\tilde{a}_{s}$ of a machine [4]. Similarly, the imaginary part of the apparent mass matrix $\tilde{M}_{r r}$ of the seating structure is less than that of the apparent mass matrix $\tilde{M}_{r s}$ between the machine and the seating structure. The imaginary part of the transfer apparent mass $M_{r s, i \neq j}$ between the $i$-th point of the machine and the $j$-th point of the seating structure, which is the element of the apparent mass matrix $\tilde{M}_{r s}$, is also less than that of the transfer apparent mass $M_{r s, i=j}$ for a linear isolation system. The transfer apparent mass $M_{r s, i \neq j}$ means the product of the loss factor of the $i$-th isolator and the structural loss factor between the $i$-th point and the $j$-th point of the machine. In general, the structural loss factor of a machine structure is less than that of an isolator if the isolator is a natural rubber. On the other hand, the point apparent mass $M_{r s, i=j}$ means the loss factor of the $i$-th isolator. Therefore, from Eq. (11), ignoring the power flow associated with the transfer apparent mass $M_{r s, i \neq j}$ in the first term and the transfer matrix $\tilde{M}_{r r}$ in the second term, the vibrational power transmitted by the $i$-th individual isolator from a machine to a seating structure is simply expressed as follows:

$$
P_{i} \cong \frac{1}{2 \omega} \operatorname{Im}\left\{\tilde{M}_{s r, i i} \tilde{a}_{s, i} \tilde{a}_{r, i}^{*}\right\}
$$

In principle this method is the most useful as only the isolator properties need be known, and $\tilde{M}_{r s, i i} \cong$ $\frac{K_{i}}{\omega^{2}}\left(1+j \eta_{i}\right)$ for an isolator of stiffness $K_{i}$. To find the total power from $n$ isolators, the contribution of each isolator calculated from Eq. (12) would have to be summed. If a machine or a seating structure is 


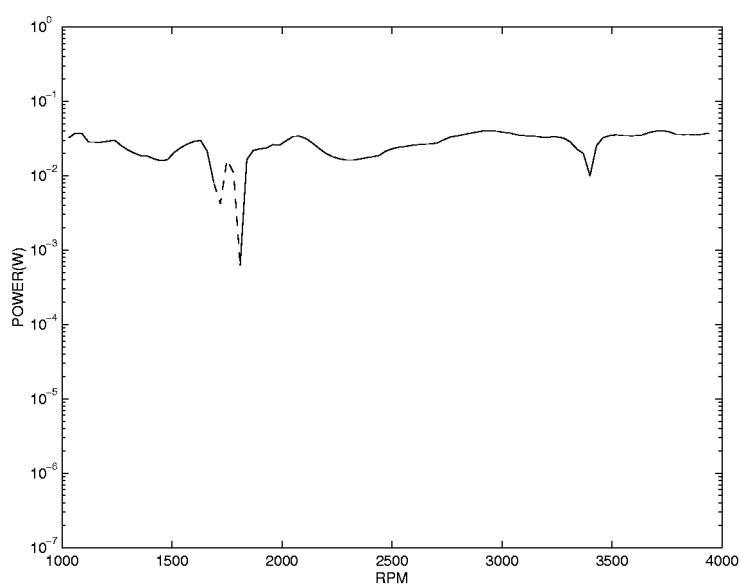

(a)

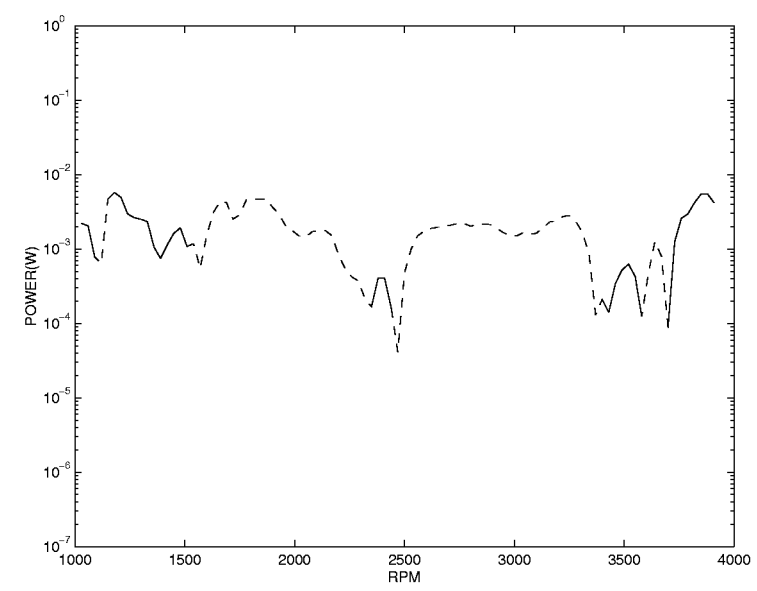

(c)

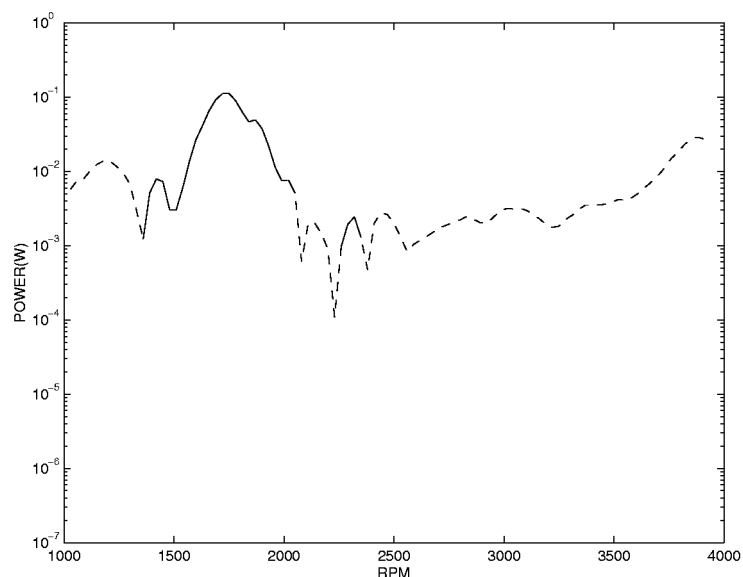

(b)

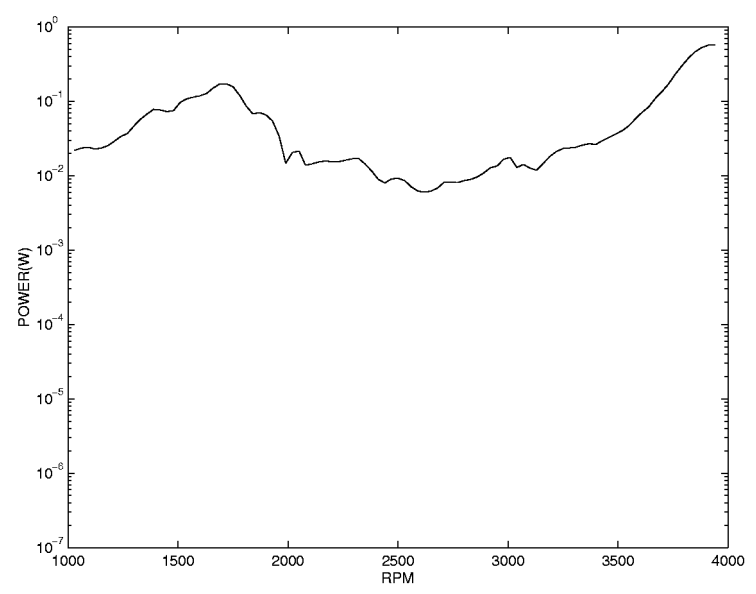

(d)

Fig. 6. Measurement of vibrational power flow through each mount (a) the engine mount (b) the front roll mount (c) the rear roll mount (d) the gear box mount; -: The positive vibrational power flow, $\cdots$ : The negative vibrational power flow.

connected with a heavy loss factor between the $i$-th isolator and the $j$-th isolator, the transfer apparent mass $M_{r s, i \neq j}$ and the transfer matrix $\tilde{M}_{r r}$ are not less than $M_{r s, i=j}$. In this case the vibrational power flow should be calculated by using Eq. (11) to remove the significant errors.

\section{Reduction of noise in compartment}

\subsection{Background of research}

A test vehicle is equipped with a $2.0 \mathrm{~L} 4$-cylinder engine and automatic transmission. The aim of this paper is to determine and improve the contribution of different transmission paths in the structure energy flow from the engine vibration to the car's interior acoustic pressure for a low frequency booming problem. The structure transmission paths under investigation can be divided in two main groups. The first group includes the direct transmission paths from the engine to the body. This yields transmission through the engine, the gearbox and the exhaust mounts. The indirect transmission can also be very important. Over the suspension triangles and shock absorbers the vibration energy from the wheels is then transmitted to the body. By a baseline test, this vehicle has interior noise booming in the back of the car at around $1800 \mathrm{rpm}$ and $2400 \mathrm{rpm}$. It can be 


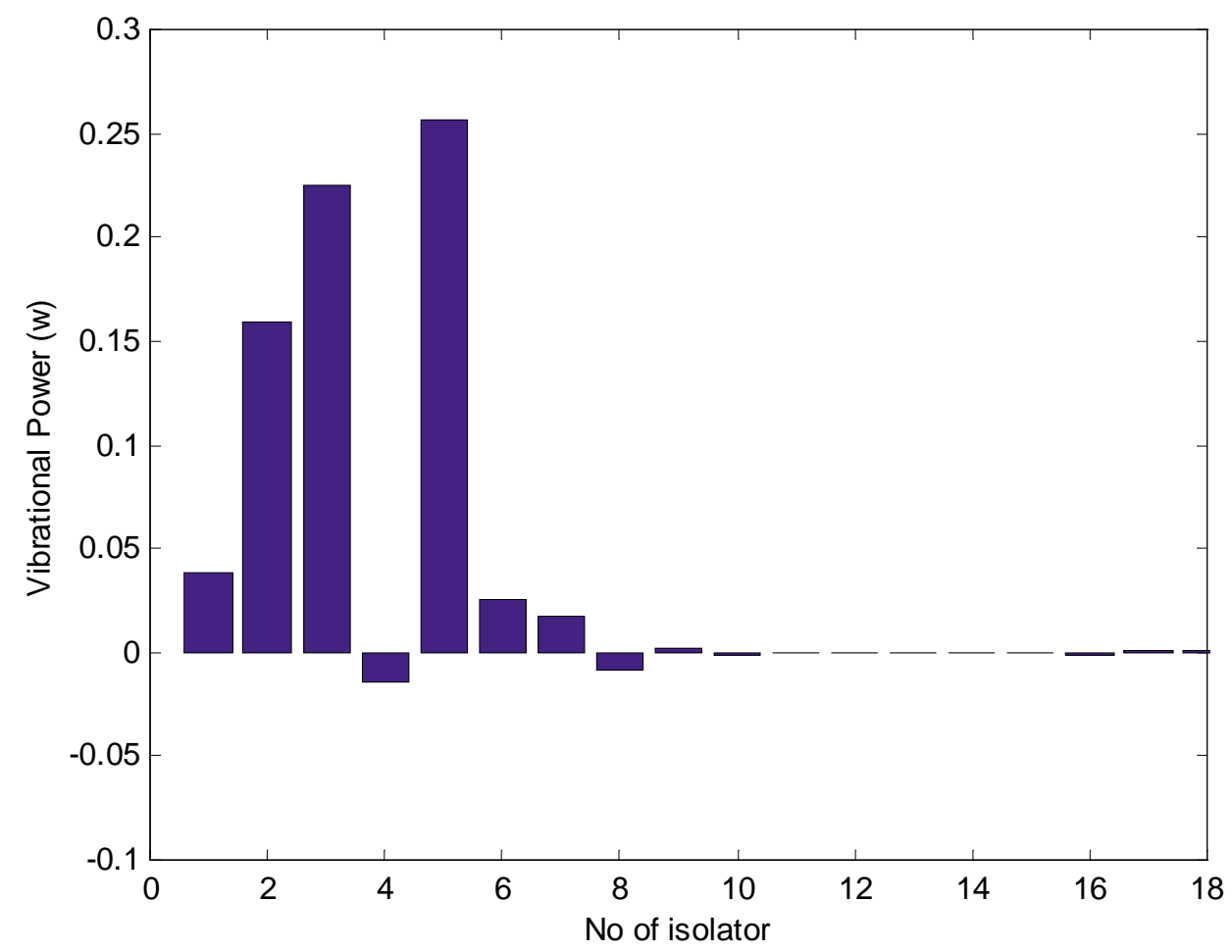

Fig. 7. Comparison between the vibrational power flow through eighteen isolators of the test vehicle.

clearly felt when the car is driven on a public road or on a rolling road in the laboratory. The frequency is the 2 nd order component of engine speed. At the first, the influence of boundary conditions on noise levels and frequency content of the interior noise and vibration has been evaluated in order to determine whether the interior noise is airborne or structure borne. This evaluation shows that the interior booming noise problem is not an airborne problem, but caused by structureborne phenomena. Therefore, a structure-borne transmission path analysis is carried out in order to identify the phenomena causing this interior booming noise.

\subsection{Application of vibrational power flow}

Figure 3 shows a test vehicle using eighteen isolators to absorb the structural energy transmitted from the engine vibration to the acoustic pressure in the compartment. In order to calculate the time-averaged vibrational power flow through each isolator using Eq. (12), the complex stiffness of each isolator is measured by using a elastomer testing system as shown in Fig. 4, and the accelerations at both sides of each isolator are measured while the test car is being driven on a chassis dynamometer in an anechoic chamber. In the elastomer testing system as shown in Fig. 4, the force excitation, provided by the hydraulic power supplier, acts on the steel block encompassing an engine mount. A rigid pin is connected to the load cell to measure the complex excitatory force. The LVDT is used for measurement of the complex displacement. Fig. 5 shows the magnitude and phase of the complex stiffness of the rear roll mount of the engine (i.e., the isolator 3 in Fig. 3) by using the elastomer testing system. This isolator does not have any resonance up to $120 \mathrm{~Hz}$. Fig. 6 shows the measured vibrational power flow through vibration isolators 1, 2, 3 and 4, which denotes the engine mount, the front roll mount and the real roll mount of the engine and the gearbox mounts, respectively. The solid line denotes the positive vibrational power flow and the dotted line denotes the negative vibrational power flow. The vibrational power flows through these four isolators are greatly higher than that through the other fourteen isolators as shown in Fig. 7. Figure 7 explains the contribution of power flows through isolators around $1800 \mathrm{rpm}$. According to this result, it is concluded that two roll mounts of the engine are important contributors to the booming noise around $1800 \mathrm{rpm}$. The rear roll mount of the engine together with the engine mount of the engine is also a contributor around $2400 \mathrm{rpm}$. 


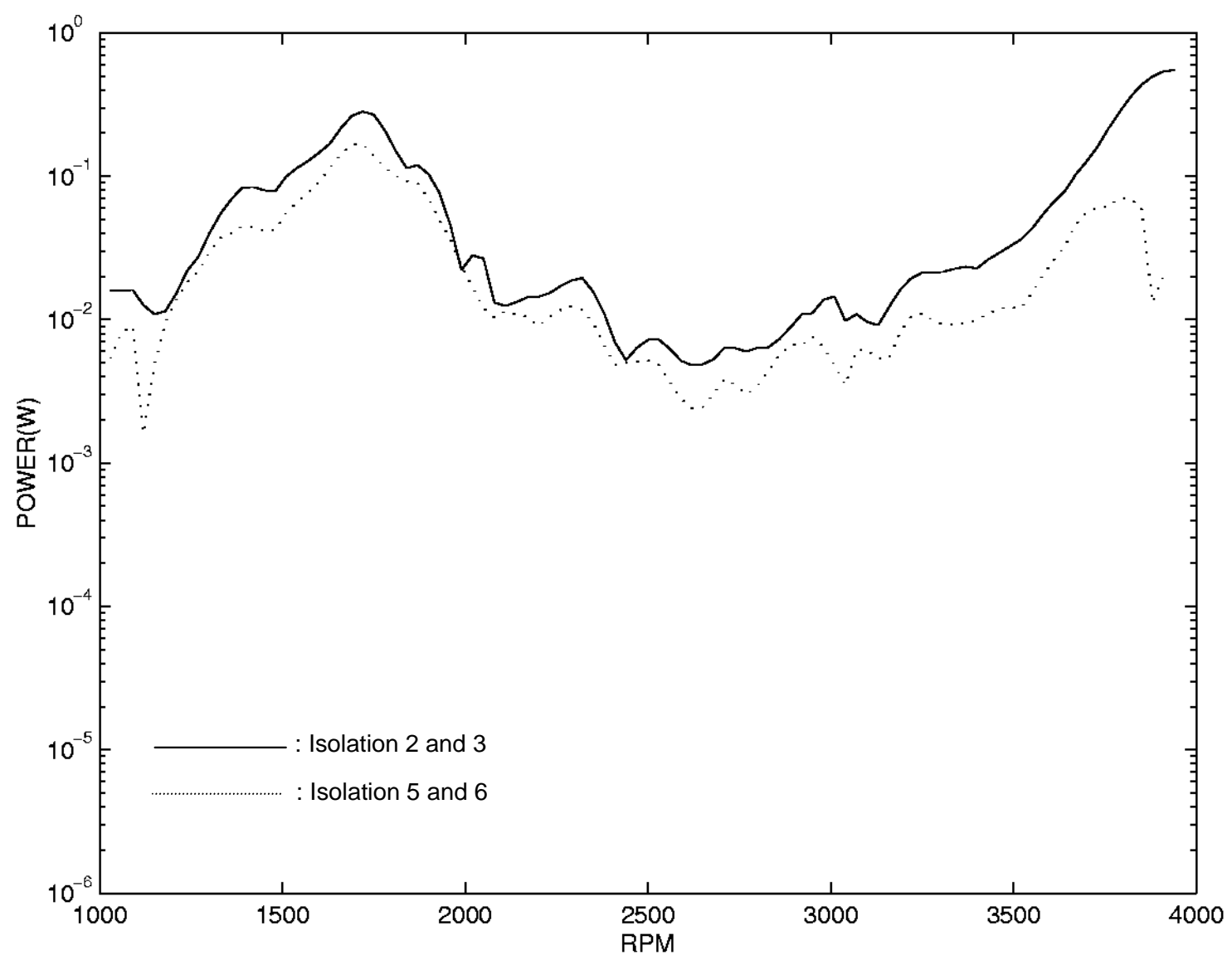

Fig. 8. Comparison between total vibrational power flow through roll two mounts of the engine and that through two bush mounts of the center member.

The gearbox mount plays a role as a sink of power flow because the vibrational power flow is negative. From Fig. 7 it can also be understood that the front bush mount of the center member of isolator 6 is also an important vibration transmission path. Therefore, it can be concluded that the vibrational power is transmitted from two roll mounts of the engine to the front bush mount of the center member. This is confirmed by comparing the total vibrational power through isolators 2 and 3 with that through isolators 5 and 6 as shown in Fig. 8. The vibrational power flows through two roll mounts of the engine and two bush mounts of the center member are nearly the same, but slightly different due to airborne noise. Therefore, in order to reduce the booming interior noise around $1800 \mathrm{rpm}$, the rear roll mount of the engine or the front bush mount of the center member should be modified. In this vehicle, there is some constraint for modification of the front bush mount. Thus, the complex dynamic stiffness of the rear roll mount is reduced by up to $15 \%$. This mod- ification yields a reduction of the interior noise level of up to 5dBA around $1800 \mathrm{rpm}$, as shown in Fig. 9. Finally, from a subjective evaluation, one cannot feel the booming noise in the compartment of the vehicle.

\section{Conclusion}

Identifying the structure-borne transmission path of energy flow from the vibration of the engine to the acoustic pressure in the compartment is useful for reducing interior noise. The estimation of the vibrational power flow through each transmission path is a useful tool for evaluating the effectiveness of vibration isolation of isolators. In this paper, a test vehicle equipped with a $2.0 \mathrm{~L} 4$-cylinder engine and automatic transmission is used. The vibrational power flow through eighteen isolators of the test vehicle has been measured. From the results, it is concluded that the vibrational power from the engine is mainly transmitted to the car 


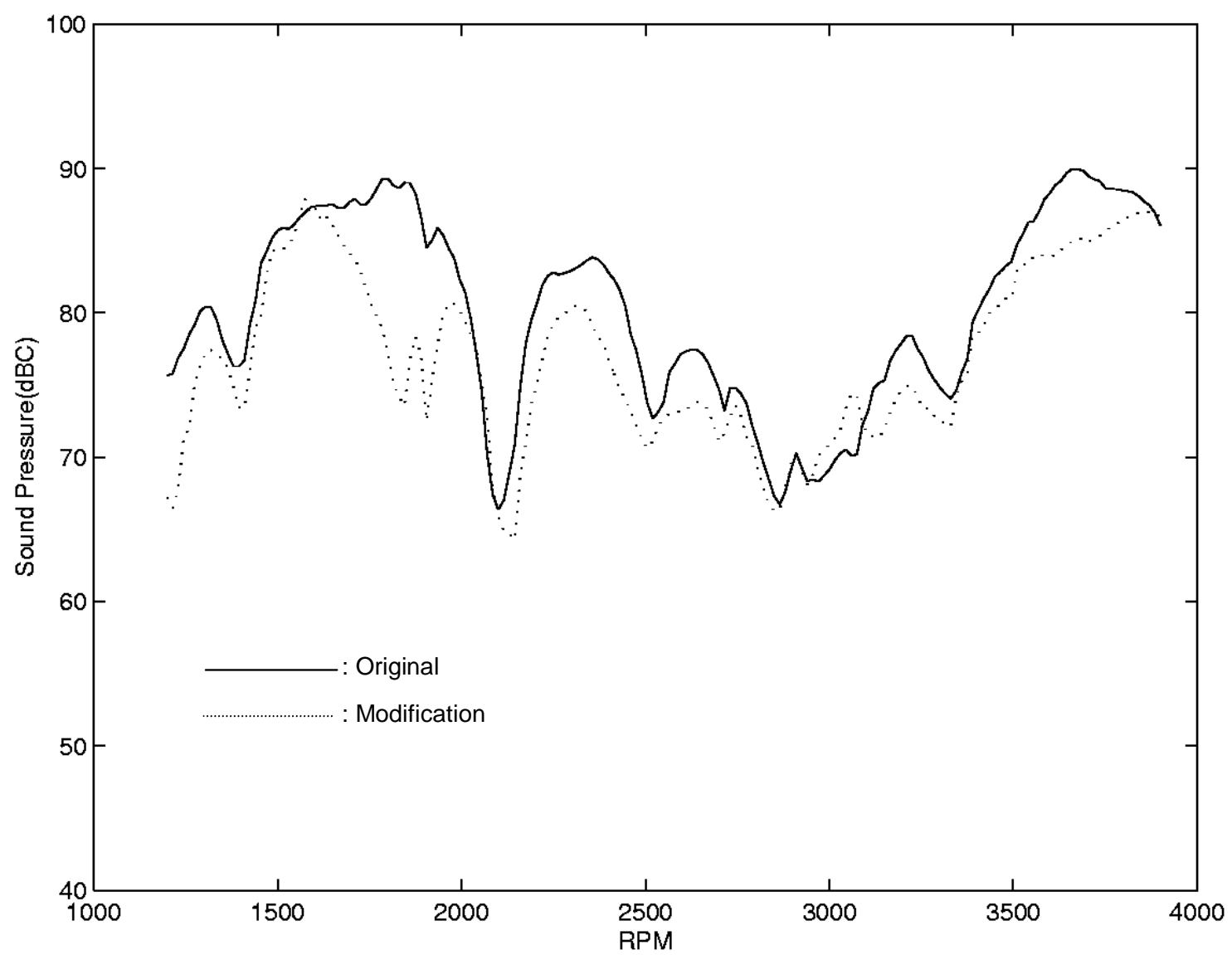

Fig. 9. Illustration of the booming noise reduction by the modification of the rear roll mount of the engine.

structure through the rear roll mount of the engine. The dynamic stiffness of the rear roll mount is reduced by $15 \%$. This modification yields a reduction of interior noise level up to 5dBA around $1800 \mathrm{rpm}$.

\section{References}

[1] H.G.D. Goyder and R.G. White, Vibrational power Flow From Machines into Builtup Structure, Part I, II, III, Journal of Sound and Vibration 68(1) (1980), 59-75.

[2] W. Hendricx and D. Vandenbroeck, Suspension Analysis in View of Road Noise Optimization, SAE 931343, 1993.

[3] B. Petersson and J. Plunt, On Effective Mobilities in the Predic- tion of Structure-Borne Sound Transmission between a Source Structure and a Receiving Structure, Part I, II, III, Journal of Sound and Vibration 82(4) (1982), 517-540.

[4] R.J. Pinnington, Power Flow Through Machine Isolators to Resonant and Non-Resonant Beams, Journal of Sound and Vibration 75(2) (1981), 179-197.

[5] R.J. Pinnington, Vibrational Power Flow Transmission to a Seating of a Vibration Isolated Motor, Journal of Sound and Vibration 118(3) (1987), 515-530.

[6] R.J. Pinnington, Transient Energy Flow Between Two Coupled Beams, Journal of Sound and Vibration 189(2) (1996), 265287.

[7] P.J.G. Van der Linden and J.K. Fun, Using Mechanical-Acoustic Reciprocity for Diagnosis of Structure Borne Sound in Vehicles, SAE 931340, 1993. 

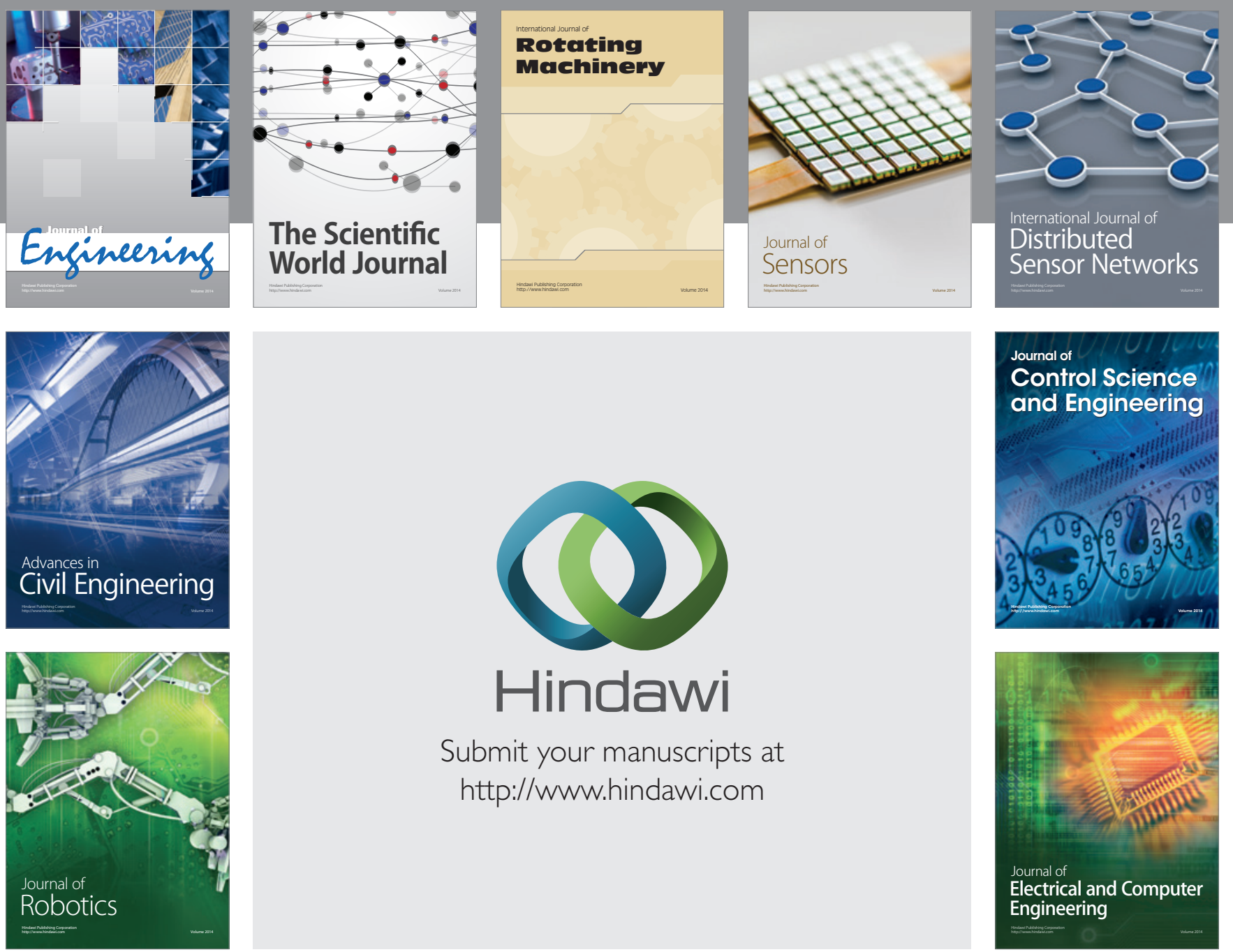

Submit your manuscripts at

http://www.hindawi.com
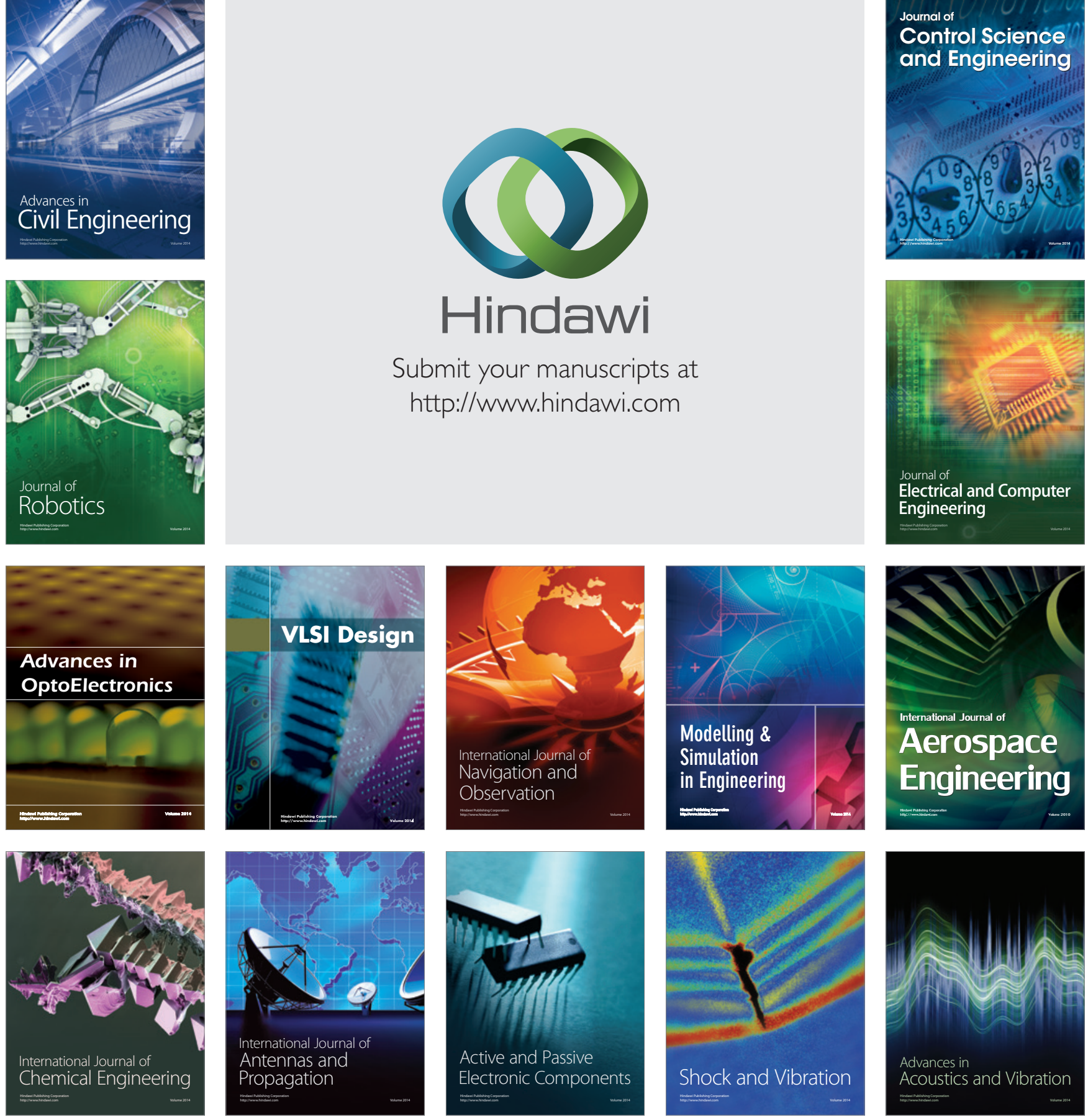